\title{
Shaping of molecular weight distribution by iterative learning probability density function control strategies
}

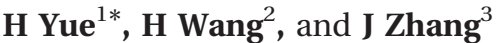 \\ ${ }^{1}$ Industrial Control Centre, University of Strathclyde, Glasgow, UK \\ ${ }^{2}$ Control Systems Centre, University of Manchester, Manchester, UK \\ ${ }^{3}$ Department of Automation, North China Electric Power University, Beijing, People's Republic of China
}

The manuscript was received on 27 February 2008 and was accepted after revision for publication on 1 May 2008.

DOI: 10.1243/09596518JSCE584

\begin{abstract}
A mathematical model is developed for the molecular weight distribution (MWD) of free-radical styrene polymerization in a simulated semi-batch reactor system. The generation function technique and moment method are employed to establish the MWD model in the form of Schultz-Zimm distribution. Both static and dynamic models are described in detail. In order to achieve the closed-loop MWD shaping by output probability density function (PDF) control, the dynamic MWD model is further developed by a linear B-spline approximation. Based on the general form of the B-spline MWD model, iterative learning PDF control strategies have been investigated in order to improve the MWD control performance. Discussions on the simulation studies show the advantages and limitations of the methodology.
\end{abstract}

Keywords: molecular weight distribution (MWD), B-spline model, probability density function (PDF), iterative learning control (ILC)

\section{INTRODUCTION}

It is widely recognized that the molecular weight distribution (MWD) of a polymer is one of the most important variables to be controlled in industrial polymerization processes because it directly affects many of the polymer's end-use properties such as thermal properties, stress-strain properties, impact resistance, strength, and hardness $[\mathbf{1}, \mathbf{2}]$. There has been much incentive to control MWD accurately during polymerization. The research into the modelling and control of MWD in polymerization has constituted an important area in process control for more than a decade, where the aim is to investigate proper control strategies that can effectively control the shape of MWD following the quality requirements of the end-use polymer.

MWD control and process optimization require on-line MWD information. With the development of hardware and software sensors for on-line monitor-

*Corresponding author: Industrial Control Centre, Department of Electronic and Electrical Engineering, University of Strathclyde, 50 George Street, Glasgow G1 1QE, UK. email: hong.yue@ eee.strath.ac.uk ing of the polymerization reactions, some polymer properties can be measured continuously or semicontinuously during operations [3]. However, information on MWD is still largely provided by mathematical models during control since the on-line MWD measurement is an unsolved issue in polymerization processes. Mechanistic MWD models can be developed under the framework of population balance, which include a set of differential equations describing the dynamics of the reaction species such as initiator, monomer, radicals, and polymers of different chain length. These equations are functions of kinetic mechanism and reaction operation conditions of the polymerization process [4]. As the polymer chain length of interest is usually a huge number up to maybe millions, direct solution of these hundreds of thousands of differential equations is infeasible in most real situations and therefore various numerical techniques have been devised to address this issue. Computational methods include the adaptive orthogonal collocation algorithm $[\mathbf{5}, \mathbf{6}]$ and the method of finite molecular weight moments [7]. Some parameterized or statistical methods are also developed for MWD description, such as Markov chain [8-10], Flory distribution 
[11-13], Stockmayer distribution [14], Weibull distribution $[15,16]$, and Schultz-Zimm distribution $[17,18]$. For many practical problems of linear polymerization under steady state or quasi-steady state conditions, the MWD of polymer chains can be described satisfactorily by the Schulz-Flory distribution $[4,19,20]$. The general way to develop this type of parameterized distribution model consists of three steps. Firstly, set up the differential equation models for reaction species. Secondly, use the generation function technique to establish the leading moments with respect to the distribution. Thirdly, obtain the parameters of the distribution function from the leading moments by optimization.

A number of control strategies have been developed to realize MWD control in batch and semibatch processes (see reviews in references [21] and [22]). These methods can generally be divided into task level control and execution level control [1]. In the task level control (first step), the optimal time profiles of process operations are determined offline aiming to attain the desired MWD at the end of the control cycle. The time profiles will be used as set point trajectories of manipulated variables, for instance reaction temperature, initiator concentration, feed rate of the monomer or chain transfer agent, etc. In order to track the optimal trajectory during operations, regulatory control is implemented in the execution level (second step), in which online techniques, such as state estimation with extended Kalman filter, are developed to update the MWD information or the manipulated control profiles $[1,21,23,24]$. Controllers from proportional-integral-derivative (PID) to model-based nonlinear control such as multivariable predictive control have been attempted in the execution level. MWD control following these two steps can be found in many examples $[\mathbf{1}, \mathbf{2}, \mathbf{2 1}, \mathbf{2 4 - 3 2}]$.

The control efforts in the above discussions should be regarded as open-loop control in terms of the MWD property because the optimal profiles relating to the desired MWD are determined in an off-line manner. To realize closed-loop control of dynamic MWD, the idea of output probability density function (PDF) control allows a unique solution. Here the controller is designed to make the output PDF follow a desired PDF [33]. The development of output PDF control was inspired by requirements from real industries, of which the system output relates to space distribution. In addition to MWD systems, examples also include particle size distribution (PSD) control in polymerization processes, [34-36] PSD control in crystallization and powder industries [37-40], fibre length distribution control in paper industries [41], flame temperature distribution control in combustion processes [42], etc. Unlike the mean and variance control in Gaussian processes, the output PDF is a function not only of time but also of a space variable. The dynamics of the output PDF can thus be generally described by a partial differential equation (PDE) in terms of both the time and space variables, which defines the PDF shape at each time instant. However, direct use of PDE models is difficult in practice in that either such a model is difficult to establish due to the complicated nature of processes or the obtained control algorithms are too complicated to be applied in real-time situations. The output PDF control strategies were proposed to solve this type of problem $[33,43,44]$, in which the main technique is the use of a set of fixed basis functions together with a group of time-varying weights to approximate the output PDF at each time instant.

Using function approximations in PDF modelling, a large number of basis functions are required when the PDF dynamics is complicated. Since the PDF of a process output can vary widely over operations, it may be unrealistic to capture the output behaviour with fixed basis functions. As a result, it would be ideal to be able to update the basis functions during the control process. This is especially suitable for systems that have a nature of repetitive closed-loop operations such as the batch-to-batch processes in chemical engineering. These batches are iterative by nature and in practice it is expected that the closed-loop performance becomes improved from batch to batch. In this context, the iterative learning control (ILC) could be very well applied $[\mathbf{4 5}, \mathbf{4 6}]$. The important aspect in ILC is to update the control input in the $k$ th batch from the control in the $(k-1)$ th batch plus a correction term that is related to the closed-loop performance of the $k$ th batch. This strategy has the advantage of improving the closed-loop system performance along with the progress of the operation $[47,48]$.

For those continuous processes that do not operate in a batch mode, the ILC principle can also be applied by dividing the control horizon into several time-domain intervals, where each interval is regarded as a pseudo batch. The main difference in the latter case is that, for the pseudo-batches in a continuous process, the initial conditions of the system states are taken from the final states in the previous batch rather than keeping the same initial conditions for all the batches. In recent years, some iterative learning PDF control strategies have been developed using B-spline basis functions $[\mathbf{4 9}, \mathbf{5 0}]$ and radial basis functions $[\mathbf{5 1}, \mathbf{5 2}]$ respectively. 
In this work, two iterative learning PDF control methods have been attempted in the closed-loop MWD control of a simulated semi-batch polymerization process. The paper is structured as follows. In section 2, development of the static and dynamic MWD models is presented. The first-principle model is then approximated by a linear B-spline model in an iterative form in section 3 . In the fourth section, the methodology of iterative learning PDF control is outlined. The simulated case study of MWD shaping by iterative learning PDF control is presented in section 5. Conclusions are given in the final section.

\section{MWD MODEL DEVELOPMENT}

\subsection{The styrene polymerization process}

The process of interest is a simulated styrene polymerization system in a semi-batch reactor. Styrene is the monomer for polymerization and azobisisobutyronitrile is the initiator. These two flows are injected into the reactor with a ratio between them that can be adjusted. The reaction temperature is assumed to be kept constant during the reaction process. The total flowrate to the system, $F$, is composed of the monomer flow, $F_{\mathrm{M}}$, and the initiator flow, $F_{\mathrm{I}}$, i.e. $F=F_{\mathrm{M}}+F_{\mathrm{I}}$. The monomer input ratio is defined as

$$
c=\frac{F_{\mathrm{M}}}{F_{\mathrm{I}}+F_{\mathrm{M}}}
$$

The output MWD will be changed when the ratio $c$ is changed. In this presented work, the monomer input ratio $c$ is selected as the control input. When the flowrate of the initiator is fixed, the change in $c$ corresponds to the change in the monomer flowrate. It is a common practice to use the flowrate of the monomer as a manipulated variable in batch or semi-batch MWD control systems [28]. The polymerization system is shown in Fig. 1.

The following free radical polymerization mechanisms are considered in the modelling:

(a) Initiation

$$
\begin{aligned}
& I \stackrel{K_{\mathrm{d}}}{\longrightarrow} 2 R^{*} \\
& R^{*}+M \stackrel{K_{\mathrm{i}}}{\longrightarrow} R_{1}
\end{aligned}
$$

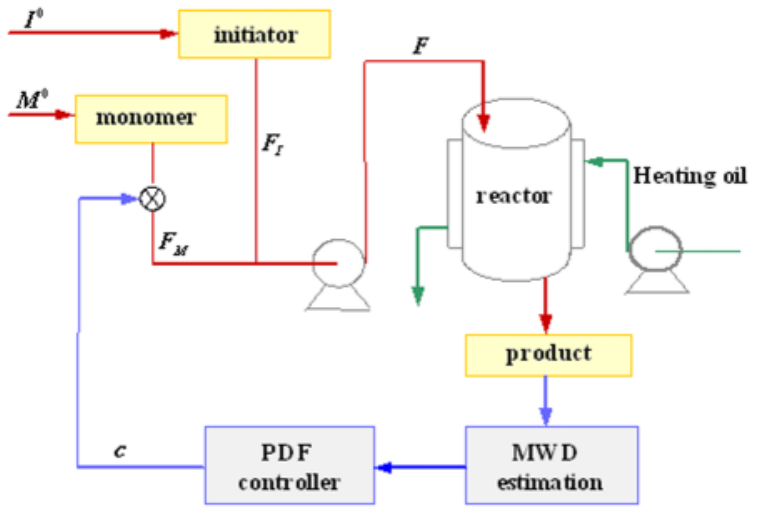

Fig. 1 Simulated semi-batch MWD control system

(b) Chain propagation

$$
R_{j}+M \stackrel{K \mathrm{p}}{\longrightarrow} R_{j+1}
$$

(c) Chain transfer to monomer

$$
R_{j}+M \stackrel{K_{\mathrm{trm}}}{\longrightarrow} P_{j}+R_{1}
$$

(d) Termination by combination

$$
R_{j}+R_{i} \stackrel{K_{\mathrm{t}}}{\longrightarrow} P_{j+i}
$$

where $I$ is the initiator, $M$ is the monomer, $R^{*}$ is the primary radical, $R_{j}$ is the live polymer radical with chain length $j, P_{j}$ is the dead polymer with chain length $j$, and $K_{\mathrm{d}}, K_{\mathrm{i}}, K_{\mathrm{p}}, K_{\mathrm{trm}}$, and $K_{\mathrm{t}}$ are reaction rate constants. The following mass balance equations are derived to describe the concentrations of the reaction species

$$
\begin{aligned}
& \frac{\mathrm{d} I}{\mathrm{~d} t}=\frac{I^{0}-I}{\theta}-K_{\mathrm{d}} I \\
& \frac{\mathrm{d} M}{\mathrm{~d} t}=\frac{M^{0}-M}{\theta}-2 K_{\mathrm{i}} I-\left(K_{\mathrm{p}}+K_{\mathrm{trm}}\right) M R \\
& \frac{\mathrm{d} R_{1}}{\mathrm{~d} t}=-\frac{R_{1}}{\theta}+2 K_{\mathrm{i}} I-K_{\mathrm{p}} M R_{1} \\
& \quad+K_{\mathrm{trm}} M\left(R-R_{1}\right)-K_{\mathrm{t}} R_{1} R
\end{aligned}
$$

$$
\begin{aligned}
\frac{\mathrm{d} R_{j}}{\mathrm{~d} t}= & -\frac{R_{j}}{\theta}-K_{\mathrm{p}} M\left(R_{j}-R_{j-1}\right)-K_{\mathrm{trm}} M R_{j} \\
& -K_{\mathrm{t}} R_{j} R \quad(j \geqslant 2)
\end{aligned}
$$




$$
\begin{aligned}
& \frac{\mathrm{d} P_{2}}{\mathrm{~d} t}=K_{\mathrm{trm}} R_{2} M+K_{\mathrm{t}} R_{1}^{2}-\frac{P_{2}}{\theta} \\
& \frac{\mathrm{d} P_{j}}{\mathrm{~d} t}=K_{\mathrm{trm}} R_{j} M+\frac{K_{\mathrm{t}}}{2} \sum_{l=1}^{j-1} R_{l} R_{j-l}-\frac{P_{j}}{\theta} \quad(j \geqslant 3)
\end{aligned}
$$

where $\theta=V / F$ ( $V$ is the volume of the reactor) is the average residential time of the reactants in the reactor and

$$
R=\sum_{j=1}^{\infty} R_{j}
$$

is the total concentration of the radicals. To use the generation function technique, denoting

$$
P=\sum_{j=2}^{\infty} P_{j}
$$

as the total concentration of the dead polymers, the following formulation can be obtained from equations (4) to (9) to give

$$
\begin{aligned}
& \frac{\mathrm{d} R}{\mathrm{~d} t}=-\frac{R}{\theta}+2 K_{\mathrm{i}} I-K_{\mathrm{t}} R^{2} \\
& \frac{\mathrm{d} P}{\mathrm{~d} t}=-\frac{P}{\theta}+K_{\mathrm{trm}} M\left(R-R_{1}\right)+\frac{K_{\mathrm{t}}}{2} R^{2}
\end{aligned}
$$

$R_{1}$ in equation (11) can be ignored compared with $R$ because of its low concentration, i.e.

$$
\frac{\mathrm{d} P}{\mathrm{~d} t}=-\frac{P}{\theta}+K_{\mathrm{trm}} M R+\frac{K_{\mathrm{t}}}{2} R^{2}
$$

\subsection{Static MWD model}

The static solutions to the concentrations of the reaction species can be derived from their dynamic equations. Denote

$$
\alpha=1+\frac{K_{\mathrm{trm}}}{K_{\mathrm{p}}}+\frac{K_{\mathrm{t}} R}{K_{\mathrm{p}} M}+\frac{1}{K_{\mathrm{p}} M \theta}
$$

By taking equations (2), (3), (10), and (12) to be zero, then

$$
I=\frac{I^{0}}{1+K_{\mathrm{d}} \theta}
$$

$$
\begin{aligned}
& R=\frac{-1 / \theta+\sqrt{1 / \theta^{2}+8 K_{\mathrm{t}} K_{\mathrm{i}} I}}{2 K_{\mathrm{t}}} \\
& M=\frac{M^{0}}{1+\left(K_{\mathrm{p}}+K_{\mathrm{trm}}\right) R \theta} \\
& P=\theta\left(K_{\mathrm{trm}} M R+\frac{K_{\mathrm{t}}}{2} R^{2}\right)
\end{aligned}
$$

Similarly, from equations (4), (5), (6), and (7), the static concentrations of radicals and polymers are

$$
\begin{aligned}
& R_{1}=\frac{2 K_{\mathrm{i}} I+K_{\mathrm{trm}} M R}{K_{\mathrm{p}} M \alpha} \\
& R_{j}=\alpha^{-1} R_{j-1}=\alpha^{-(j-1)} R_{1} \quad(j \geqslant 2) \\
& P_{2}=\theta\left(K_{\mathrm{trm}} M R_{2}+K_{\mathrm{t}} R_{1}^{2}\right) \\
& P_{j}=\theta\left(K_{\mathrm{trm}} M R_{j}+\frac{K_{\mathrm{t}}}{2} \sum_{l=1}^{j-1} R_{l} R_{j-l}\right) \quad(j \geqslant 3)
\end{aligned}
$$

Substituting equation (19) into (20) and (21) and divided by $P$, the normalized MWD at static state can be obtained to be

$$
P_{j}= \begin{cases}\frac{\theta}{P}\left(\alpha^{-1} K_{\mathrm{trm}} M R_{1}+K_{\mathrm{t}} R_{1}^{2}\right) & j=2 \\ \frac{\theta}{P}\left(\alpha^{-(j-1)} K_{\mathrm{trm}} M R_{1}\right. & \\ \left.+\frac{j-1}{2} \alpha^{-(j-2)} K_{\mathrm{t}} R_{1}^{2}\right) & j \geqslant 3\end{cases}
$$

It can be seen from equation (22) that

$$
\sum_{j=2}^{\infty} P_{j}=1
$$

Therefore, the static MWD can be taken as a discrete PDF of the chain length $j$.

\subsection{Dynamic MWD model}

For the dynamic MWD model, the distribution of $P_{j}$ is not only a function of the chain length but also a 
function of time. At each time instance, the moment method is used for the MWD description.

The moments of the number chain-length distributions of radicals and polymers are defined as

$$
\begin{aligned}
& U_{k}=\sum_{j=1}^{+\infty} j^{k} R_{j}, \quad k=0,1,2, \ldots \\
& Z_{k}=\sum_{j=2}^{+\infty} j^{k} P_{j}, \quad k=0,1,2, \ldots
\end{aligned}
$$

It can be seen from equations (8) and (9) that $U_{0}=R$ and $Z_{0}=P$. For the radicals, the differential equations of the leading moments are derived using the generation function technique as follows

$$
\begin{aligned}
\frac{\mathrm{d} U_{0}}{\mathrm{~d} t}= & -\frac{U_{0}}{\theta}+2 K_{\mathrm{i}} I-K_{\mathrm{t}} U_{0}^{2} \\
\frac{\mathrm{d} U_{1}}{\mathrm{~d} t}= & -\frac{U_{1}}{\theta}+2 K_{\mathrm{i}} I+K_{\mathrm{p}} U_{0} M-K_{\mathrm{t}} U_{0} U_{1} \\
& +K_{\mathrm{trm}} M\left(U_{0}-U_{1}\right) \\
\frac{\mathrm{d} U_{2}}{\mathrm{~d} t}= & -\frac{U_{2}}{\theta}+2 K_{\mathrm{i}} I+K_{\mathrm{p}} M\left(2 U_{1}+U_{0}\right) \\
& -K_{\mathrm{t}} U_{0} U_{2}+K_{\mathrm{trm}} M\left(U_{0}-U_{2}\right)
\end{aligned}
$$

Similarly, the three leading moments of the polymers are derived to be

$$
\begin{aligned}
& \frac{\mathrm{d} Z_{0}}{\mathrm{~d} t}=-\frac{Z_{0}}{\theta}+K_{\mathrm{trm}} M U_{0}+\frac{K_{\mathrm{t}}}{2} U_{0}^{2} \\
& \frac{\mathrm{d} Z_{1}}{\mathrm{~d} t}=-\frac{Z_{1}}{\theta}+K_{\mathrm{trm}} M U_{1}+K_{\mathrm{t}} U_{0} U_{1} \\
& \frac{\mathrm{d} Z_{2}}{\mathrm{~d} t}=-\frac{Z_{2}}{\theta}+K_{\mathrm{trm}} M U_{2}+K_{\mathrm{t}} U_{0} U_{2}+K_{\mathrm{t}} U_{1}^{2}
\end{aligned}
$$

The mean and variance of the MWD are related to the moments by

$$
\begin{aligned}
& \mu=\frac{\sum_{j=2}^{+\infty} j P_{j}}{\sum_{j=2}^{+\infty} P_{j}}=\frac{Z_{1}}{Z_{0}} \\
& \sigma^{2}=\frac{\sum_{j=2}^{+\infty}(j-\mu)^{2} P_{j}}{\sum_{j=2}^{+\infty} P_{j}}=\frac{Z_{2}}{Z_{0}}-\frac{Z_{1}^{2}}{Z_{0}^{2}}
\end{aligned}
$$

Theoretically, an exact formulation of MWD requires countless number of moments, which is infeasible in practical computation. An alternative method is to choose a distribution function to approximate the real MWD. For the polymer discussed in this work, the well-known Schultz-Zimm distribution is considered to be suitable for the MWD description. With this function, a simple analytical expression for the scattering from the distribution is available. The normalized Schultz-Zimm distribution is defined as [53]

$$
f(n)=\frac{h^{h} n^{h-1} \exp \left(-h n / M_{n}\right)}{M_{n}^{h} \Gamma(h)} \quad(n \geqslant 1)
$$

where $n$ is the chain length, $h$ is a parameter indicating the distribution breadth, $M_{n}$ is the number average chain length which is defined as $M_{n}=Z_{1} / Z_{0}$, and $\Gamma$ is the gamma function defined as $\Gamma(h)=\int_{0}^{\infty} n^{h-1} \mathrm{e}^{-n} \mathrm{~d} n$. When $h=1$, the SchultzZimm distribution reduces to the exponential Flory distribution, which is another commonly used distribution for MWD. The mean and variance of the Schultz-Zimm distribution are

$$
\begin{aligned}
\mu & =\int_{0}^{\infty} n f(n) \mathrm{d} n=\int_{0}^{\infty} \frac{\left.h^{h} n^{h} \exp \left(-h n / M_{n}\right)\right)}{M_{n}^{h} \Gamma(h)} \mathrm{d} n \\
& =M_{n} \\
\sigma^{2} & =\int_{0}^{\infty}(n-\mu)^{2} f(n) \mathrm{d} n=\frac{h+1}{h} M_{n}^{2}-\mu^{2}
\end{aligned}
$$

By comparing equations (32) and (33) with (35) and (36), the two parameters of the Schultz-Zimm distribution are found to be

$$
\begin{aligned}
& h=\frac{Z_{1}^{2}}{Z_{0} Z_{2}-Z_{1}^{2}} \\
& M_{n}=Z_{1} / Z_{0}
\end{aligned}
$$

The first-principle model describes the entire MWD by a Schultz-Zimm distribution. From the model development process, it can be seen that the parameters of the distribution function are linked to the dynamic process via moments of polymers and concentrations of reaction species.

The calculation of the dynamic MWD can be summarized in the following three steps:

1. Obtain $Z_{0}, Z_{1}, Z_{2}$ from equations (2), (3), and (26) to $(31)$.

2. Obtain $h$ and $M_{n}$ from equations (37) and (38). 
3. Formulate the MWD by equation (34).

\section{B-SPLINE APPROXIMATION FOR ITERATIVE LEARNING CONTROL}

Though the first-principle model has the ability of predicting MWD dynamically, it will not be used directly in MWD control. Instead, a B-spline approximation model is established from the data produced by the first-principle model. The reason for doing so is that rather than taking the MWD control as a specific problem, it is more general to treat it as a problem with the output being a function of time and space variables. Also, when using a general Bspline model, even if the first-principle model is not available, the PDF control strategy can still be applied with the measurement of MWD if online techniques permit. Here the well-established Bspline PDF model is redeveloped to suit the purpose of iterative learning PDF control in the MWD system.

\subsection{Output PDF model with fixed B-spline functions}

Consider a continuous PDF $\gamma_{i}(y)$ defined on the $[a, b]$ interval. The linear B-spline neural network can be used to give an approximation of $\gamma_{i}(y)$ [33]

$$
\gamma_{i}(y)=\sum_{l=1}^{n} \omega_{l}\left(u_{i}\right) B_{l}(y)+e_{0}
$$

where the subscript $i$ indicates the $i$ th sample time. Throughout the paper, $\gamma_{i}(y)$ is assumed to be measurable, $u_{i}$ is the control input at time $i(i=1$, $2, \ldots, m), m$ is the total sample number along the time axis, $B_{l}(y)(l=1, \ldots, n)$ are the pre-specified basis functions defined on the interval of $y \in[a, b], n$ is the total number of the basis functions used for the approximation to $\gamma_{i}(y), \omega_{l}\left(u_{i}\right)(l=1, \ldots, n)$ are the expansion weights, and $e_{0}$ represents the approximation error which satisfies $|e|<\delta_{1}\left(\delta_{1}\right.$ is a known small positive number). To simplify the expression, $e_{0}$ is neglected in the following. Due to the fact that the integration of a PDF over its definition domain should be 1 , there are only $n-1$ independent weights out of the original $n$ weights [44]. Using this B-spline approximation and considering linear dynamics in the weights vector, the following discrete output PDF model is formulated

$$
\boldsymbol{V}_{i}=\overline{\mathbf{A}} \boldsymbol{V}_{i-1}+\overline{\mathbf{B}} u_{i-1}
$$

$$
\gamma_{i}(y)=\mathbf{C}(y) \boldsymbol{V}_{i}+L(y)
$$

where $\boldsymbol{V}_{i}=\left[\omega_{1}\left(u_{i}\right), \omega_{2}\left(u_{i}\right), \ldots, \omega_{n-1}\left(u_{i}\right)\right]^{\mathrm{T}}$ is the weights vector of the B-spline model at time $i . \overline{\mathbf{A}}$ and $\overline{\mathbf{B}}$ are the system parameter matrices of proper dimensions. $\mathbf{C}(y)$ and $L(y)$ are related to the B-spline functions by

$$
\begin{aligned}
\varphi_{l} & =\int_{a}^{b} B_{l}(y) \mathrm{d} y, \quad l=1, \ldots, n \\
L(y) & =B_{n}(y) / \varphi_{n} \\
c_{r}(y) & =B_{r}(y)-L(y) \varphi_{r}, \quad r=1, \ldots, n-1 \\
\mathbf{C}(y) & =\left[c_{1}(y), c_{2}(y), \ldots, c_{n-1}(y)\right]
\end{aligned}
$$

\subsection{B-spline model in iterative form}

In order to formulate the ILC law for batch-to-batch PDF control, the system model is written as

$$
\begin{gathered}
\boldsymbol{V}_{k, i}=\overline{\mathbf{A}}_{k} \boldsymbol{V}_{k, i-1}+\overline{\mathbf{B}}_{k} u_{k, i-1} \\
\gamma_{k, i}(y)=\mathbf{C}_{k}(y) \boldsymbol{V}_{k, i}+L_{k}(y)
\end{gathered}
$$

where the added subscript $k$ indicates the $k$ th batch and $\gamma_{k, i}(y), \boldsymbol{V}_{k, i}$, and $u_{k, i}$ are the output PDF, weights vector, and control input respectively at the $i$ th sample time in the $k$ th batch. For the $k$ th batch, $\mathbf{C}_{k}(y)$ and $L_{k}(y)$ are formulated to be

$$
\begin{aligned}
& \varphi_{k, l}=\int_{a}^{b} B_{k, l}(y) \mathrm{d} y, \quad l=1, \ldots, n \\
& L_{k}(y)=B_{k, n}(y) / \varphi_{k, n} \\
& c_{k, r}(y)=B_{k, r}(y)-L_{k}(y) \varphi_{k, r}, \quad r=1, \ldots, n-1 \\
& \mathbf{C}_{k}(y)=\left[c_{k, 1}(y), \quad c_{k, 2}(y), \ldots, \quad c_{k, n-1}(y)\right]
\end{aligned}
$$

The input-output expansion of (42) and (43) is as follows

$$
\begin{aligned}
f_{k, i}(y) & =\gamma_{k, i}(y)-L_{k}(y) \\
& =\sum_{p=1}^{n-1} \alpha_{k, p} f_{k, i-p}(y)+\sum_{q=0}^{n-2} \mathbf{C}_{k}(y) \mathbf{D}_{k, q} u_{k, i-1-q}
\end{aligned}
$$


with $\mathbf{D}_{k, q}=\left[\begin{array}{llll}d_{k, q, 1}, & d_{k, q, 2}, \ldots, d_{k, q, n-1}\end{array}\right]^{\mathrm{T}}$, which are directly related to matrices $\overline{\mathbf{A}}_{k}$ and $\overline{\mathbf{B}}_{k}$.

Denoting

$$
\begin{aligned}
\theta_{k}= & {\left[\alpha_{k, 1}, \ldots, \alpha_{k, n-1}, d_{k, 0,1}, \ldots, d_{k, 0, n-1}, \ldots,\right.} \\
& \left.d_{k, n-2,1}, \ldots, d_{k, n-2, n-1}\right] \\
\phi_{k, i}(y)= & {\left[f_{k-1, i-1}(y), \ldots, f_{k-1, i-n+1}(y),\right.} \\
& c_{k, 1}(y) u_{k-1, i-1}, \ldots, c_{k, n-1}(y) u_{k-1, i-1}, \ldots, \\
& \left.c_{k, 1}(y) u_{k-1, i-n+1}, \ldots, c_{k, n-1}(y) u_{k-1, i-n+1}\right]
\end{aligned}
$$

equation (48) can be further expressed by

$$
f_{k, i}(y)=\phi_{k, i}(y) \theta_{k}^{\mathrm{T}}
$$

This is similar to the standard form of a linear system whose parameters in $\theta_{k}$ can be estimated by leastsquares identification methods.

Within each batch, such a PDF model has been fully explained in some previous work $[33,43,44]$. However, different from the model with fixed Bsplines, the iterative model features different basis functions for each batch, which means that the Bspline functions for the PDF approximation can be updated iteratively from batch to batch in order to improve the modelling accuracy. This is important for real systems modelling where the process is normally in a time-varying and uncertain environment. For the linear weights model used in this work, the iterative update of the B-spline model also helps to address the system's non-linear nature to some extent.

\subsection{Parameter re-estimation for the updated B-spline functions}

In the iterative learning PDF control cycle, the Bspline basis functions are updated from batch to batch (this will be further explained in the next section). In each batch, when the updated basis functions are taken for PDF approximation, the model parameters in (49) need to be re-estimated.

At each sample time $i$ in the $k$ th batch, $f_{k, i}(y)$ is a continuous function of $y$ defined on $[a, b]$. Assume that $f_{k, i}(y)$ can be represented by $N$ sample points; then for each sample point $j$ there is

$$
f_{k, i}\left(y_{j}\right)=\phi_{k, i}\left(y_{j}\right) \theta_{k}^{\mathrm{T}}, \quad j=1, \ldots, N
$$

As a result, with respect to the new index $j$, the following least-squares algorithm can be used to estimate $\theta_{k}$ based on the measured output PDFs, the control inputs in the $(k-1)$ th batch, and the adjusted
B-spline functions

$$
\begin{aligned}
& \hat{\theta}_{k}(j+1)=\hat{\theta}_{k}(j)+\frac{\overline{\mathbf{P}}(j) \phi_{k, i}^{\mathrm{T}}\left(y_{j}\right) \varepsilon_{k}(j)}{1+\phi_{k, i}\left(y_{j}\right) \overline{\mathbf{P}}(j) \phi_{k, i}^{\mathrm{T}}\left(y_{j}\right)} \\
& \varepsilon_{k}(j)=f_{k, i}\left(y_{j}\right)-\phi_{k, i}\left(y_{j}\right) \hat{\theta}_{k}(j) \\
& \overline{\mathbf{P}}(j+1)=\left(I-\frac{\overline{\mathbf{P}}(j) \phi_{k, i}^{\mathrm{T}}\left(y_{j}\right) \phi_{k, i}\left(y_{j}\right)}{1+\phi_{k, i}\left(y_{j}\right) \overline{\mathbf{P}}(j) \phi_{k, i}^{\mathrm{T}}\left(y_{j}\right)}\right) \overline{\mathbf{P}}(j)
\end{aligned}
$$

where $\overline{\mathbf{P}}(1)=10^{3-6} \mathbf{I}_{(n-1) \times n}$. The recursive loops of the identification algorithm is shown in Fig. 2, in which the inner loop operation is called 'scanning' because the variable $y_{j}$ goes through the whole definition interval of $[a, b]$ by $j=1, \ldots, N$.

\section{ITERATIVE LEARNING PDF CONTROL}

\subsection{Iterative learning algorithm I}

\subsubsection{Iterative update of the B-spline model}

The sketch of the iterative learning PDF control is shown in Fig. 3, in which $u$ is the control input, $\gamma(y, u)$ is the output PDF, $g(y)$ is the target PDF, $\delta(y)$ measures the distance between the output PDF and the target PDF. This algorithm consists of three steps:

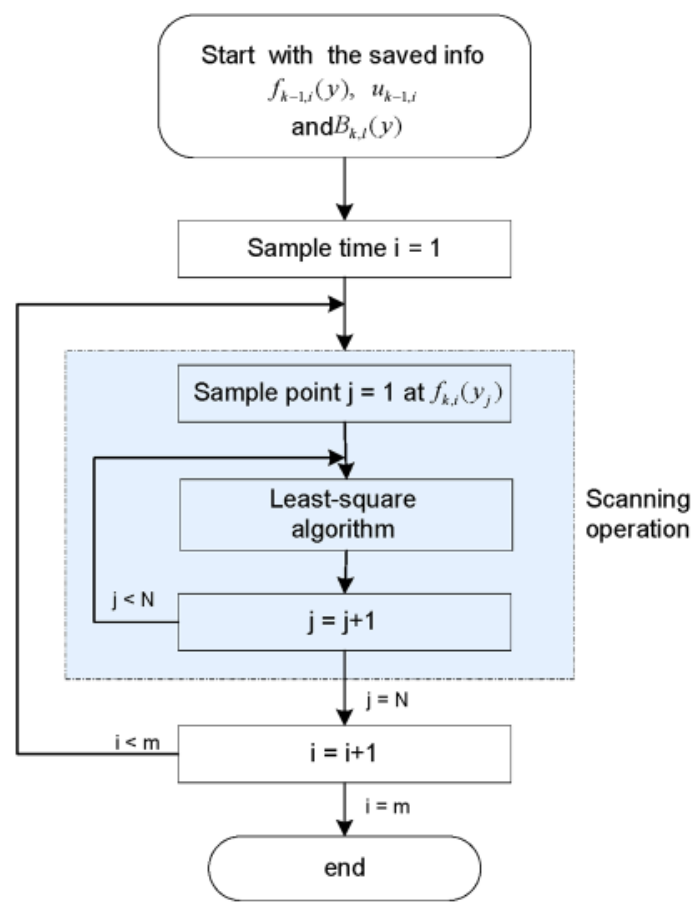

Fig. 2 The algorithm of scanning parameter identification 


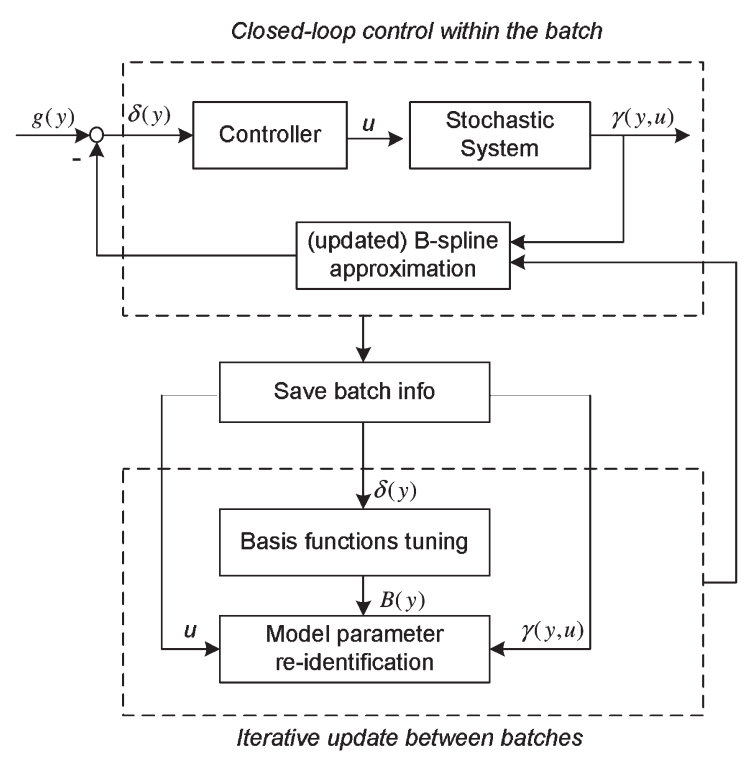

Fig. 3 Iterative learning PDF control scheme

1. Update the B-spline basis functions from the PDF control error in the previous batch.

2. Re-estimate the parameters of the B-spline model with the updated basis functions.

3. Design the PDF controller using the updated B-spline model and apply it to the current batch.

In this paper, the following polynomial B-spline is used to approximate the output PDF

$$
\begin{aligned}
& B_{k, l}(y)= \\
& \begin{cases}-\frac{4 h_{k, l}}{w_{k, l}^{2}}\left(y-y_{l 0}-\frac{w_{k, l}}{2}\right)^{2}+h_{k, l}, & y \in\left[y_{l 0}, y_{l 0}+w_{k, l}\right] \\
0, & \text { otherwise }\end{cases}
\end{aligned}
$$

for $l=1, \ldots, n$. Here $h_{k, l}$ and $w_{k, l}$ stand for the height and width of each B-spline basis function with $B_{k, l}(y) \geqslant 0, h_{k, l} \geqslant 0$ and $w_{k, l} \geqslant 0$, and $\left[y_{l 0}, y_{l 0}+w_{k, l}\right]$ is the $l$ th subinterval within $[a, b]$. As the width of the basis functions are tuned between batches, the subinterval $\left[y_{l 0}, y_{l 0}+w_{k, l}\right]$ will shift within $[a, b]$ batch by batch. It can be seen that $h_{k, l}$ and $w_{k, l}$ determine the shape of the basis functions.

Substituting (54) into equations (44), (45), and (46) gives

$$
\begin{aligned}
\varphi_{k, l} & =\int_{a}^{b} B_{k, l}(y) \mathrm{d} y \\
& =\int_{a_{k, l}}^{b_{k, l}}\left[-\frac{4 h_{k, l}}{w_{k, l}^{2}}\left(y-y_{l 0}-\frac{w_{k, l}}{2}\right)^{2}+h_{k, l}\right] \mathrm{d} y
\end{aligned}
$$

for $l=1, \ldots, n$

$$
\begin{gathered}
L_{k}(y)=\frac{-\left(4 h_{k, n} / w_{k, n}^{2}\right)\left(y-y_{n 0}-w_{k, n} / 2\right)^{2}+h_{k, n}}{\varphi_{k, n}}(56) \\
c_{k, r}(y)=-\frac{4 h_{k, r}}{w_{k, r}^{2}}\left(y-y_{r 0}-\frac{w_{k, r}}{2}\right)^{2}+h_{k, r} \\
-L_{k}(y) \varphi_{k, r}, \quad r=1, \ldots, n-1
\end{gathered}
$$

where $a_{k, l}$ and $b_{k, l}$ are the lower and upper bounds for the $l$ th B-spline basis function in the $k$ th batch and $a_{k, l}=y_{l 0}, b_{k, l}=\min \left(y_{l 0}+w_{k, l}, b\right)$. When $y \in\left[a_{k, l}\right.$, $b_{k, l}$, , the inequality $B_{k, l}(y) \geqslant 0$ is assured.

The iterative update of the basis functions are based on all the PDF tracking errors collected at each sample time from the previous batch. Denote the 2norm PDF tracking error at sample time $i$ in the $(k-$ 1)th batch as

$$
\delta_{k-1, i}=\int_{a}^{b}\left[\gamma_{k-1, i}(y)-g(y)\right]^{2} \mathrm{~d} y
$$

where $g(y)$ is the target PDF. It can be seen that $\delta_{k-1, i}$ $\geqslant 0$. The error vector that groups all the $m$ sampling errors in the $(k-1)$ th batch can be represented by

$$
\boldsymbol{E}_{k-1}=\left[\delta_{k-1,1}, \delta_{k-1,2}, \ldots, \delta_{k-1, m}\right]^{\mathrm{T}}
$$

For the B-spline basis functions defined in (54), the following P-type iterative learning law is adopted to adjust the parameters $h_{k, l}$ and $w_{k, l}$ so as to tune the shape of the functions

$$
\begin{aligned}
& \boldsymbol{H}_{k}=\boldsymbol{H}_{k-1}+\mathbf{Q}_{H} \boldsymbol{E}_{k-1} \\
& \boldsymbol{W}_{k}=\boldsymbol{W}_{k-1}+\mathbf{Q}_{W} \boldsymbol{E}_{k-1}
\end{aligned}
$$

where $\boldsymbol{H}_{k}=\left[h_{k, 1}, \ldots, h_{k, n}\right]^{\mathrm{T}}$ and $\boldsymbol{W}_{k}=\left[w_{k, 1}, \ldots, w_{k, n}\right]^{\mathrm{T}}$ are the vectors composed of the height and the width of each B-spline basis function in the $k$ th $(k \geqslant 2)$ batch. $\mathbf{Q}_{H}$ and $\mathbf{Q}_{W}$ are the learning rate matrices to be determined.

Algorithm (58) to (61) shows that at each tuning interval, the basis functions are updated using the PDF tracking errors collected from the previous batch. In addition, it can be seen from equations (58) and (59) that all the variables in $\boldsymbol{E}_{k-1}$ are always nonnegative. This shows that the learning rates $\mathbf{Q}_{H}$ and $\mathbf{Q}_{W}$ can be either positive or negative, which allows the height and the width of the B-spline basis 
functions to either increase or decrease after the tuning via equations (60) and (61). Once the basis functions are updated, the parameters of the Bspline model will be re-estimated, as presented in section 3.3.

The PDF controller is designed with the updated model by optimizing the following quadratic performance function

$$
J_{k, i}=\int_{a}^{b}\left[\gamma_{k, i}(y)-g(y)\right]^{2} \mathrm{~d} y+u_{k, i} R u_{k, i}^{\mathrm{T}}
$$

where $R>0$ is a pre-specified weighting factor. Taking $\partial J_{k, i} / \partial u_{k, i}=0$, the control input is developed to be

$$
u_{k, i}=\frac{\int_{a}^{b} \mathbf{C}_{k}(y) \mathbf{D}_{k, 0} \tilde{\boldsymbol{g}}_{k, i}(y) \mathrm{d} y}{\left.\int_{a}^{b}\left[\mathbf{C}_{k}(y) \mathbf{D}_{k, 0}\right)\right]^{2} \mathrm{~d} y+R}
$$

where

$$
\begin{aligned}
\tilde{g}_{k, i}(y)= & -\sum_{p=2}^{n-1}\left[\alpha_{k, p} f_{k, i-p+1}(y)+\mathbf{C}_{k}(y) \mathbf{D}_{k, p-1} u_{k, i-p+1}\right] \\
& -L_{k}(y)+g(y)-\alpha_{k, 1} f_{k, i}(y)
\end{aligned}
$$

is a known term at the $i$ th time instance in the $k$ th batch.

\subsubsection{Convergence analysis}

In order to guarantee the convergence of the proposed iterative learning algorithn for PDF control, appropriate learning rates in equations (60) and (61) should be chosen to tune the B-spline basis functions so that the controller can progressively improve the tracking performance batch by batch, which eventually leads to a satisfactory output PDF tracking with respect to the desired PDF. For this purpose, the following two aspects are considered to formulate some guidelines to the selection of the learning rates:

1. The weight and height of all the basis functions should always be kept non-negative, i.e. $h_{k, l} \geqslant 0$ and $w_{k, l} \geqslant 0, l=1, \ldots, n$. This constraint comes from the definition of the B-spline functions in (54).

2. For a gradually improved iterative learning algorithm, the sum of the tracking errors should be reduced batch by batch, i.e.

$$
0<\frac{\sum_{i=1}^{m} \delta_{k+1, i}}{\sum_{i=1}^{m} \delta_{k, i}} \leqslant 1
$$

\section{Denoting}

$$
\Delta_{k}=\sum_{i=1}^{m} \delta_{k, i}
$$

the inequality (64) can be expressed as

$$
0<\frac{\Delta_{k+1}}{\Delta_{k}} \leqslant 1
$$

The above sufficient conditions guarantee that the iterative leaning rules (60) and (61) are convergent and that the PDF tracking error decreases batch by batch. Assuming that the learning increments $\mathbf{Q}_{H} \mathbf{E}_{k}$ and $\mathbf{Q}_{W} \mathbf{E}_{k}$ are small, the following first-order approximation can be made

$$
\begin{aligned}
\Delta_{k+1} \approx & \Delta_{k}+\left(\left.\frac{\partial \Delta}{\partial \boldsymbol{H}}\right|_{\boldsymbol{H}=\boldsymbol{H}_{k}, \boldsymbol{W}=\boldsymbol{w}_{k}}\right)^{\mathrm{T}} \cdot \mathbf{Q}_{H} \boldsymbol{E}_{k} \\
& +\left(\left.\frac{\partial \Delta}{\partial \boldsymbol{W}}\right|_{\boldsymbol{H}=\boldsymbol{H}_{k}, \boldsymbol{W}=\boldsymbol{W}_{k}}\right)^{\mathrm{T}} \cdot \mathbf{Q}_{W} \boldsymbol{E}_{k}
\end{aligned}
$$

This leads to

$$
\begin{array}{r}
\frac{\Delta_{k+1}}{\Delta_{k}} \approx 1+\frac{\left(\partial \Delta /\left.\partial \boldsymbol{H}\right|_{\boldsymbol{H}=\boldsymbol{H}_{k}, \boldsymbol{W}=\boldsymbol{W}_{k}}\right)^{\mathrm{T}} \cdot \mathbf{Q}_{H} \boldsymbol{E}_{k}}{\Delta_{k}} \\
+\frac{\left(\partial \Delta /\left.\partial \boldsymbol{W}\right|_{\boldsymbol{H}=\boldsymbol{H}_{k}, \boldsymbol{W}=\boldsymbol{W}_{k}}\right)^{\mathrm{T}} \cdot \mathbf{Q}_{W} \boldsymbol{E}_{k}}{\Delta_{k}}
\end{array}
$$

Taking (68) into (66) yields

$$
\begin{aligned}
0<1 & +\frac{\left(\partial \Delta /\left.\partial \boldsymbol{H}\right|_{\boldsymbol{H}=\boldsymbol{H}_{k}, \boldsymbol{W}=\boldsymbol{W}_{k}}\right)^{\mathrm{T}} \cdot \mathbf{Q}_{H} \boldsymbol{E}_{k}}{\Delta_{k}} \\
+ & \frac{\left(\partial \Delta /\left.\partial \boldsymbol{W}\right|_{\boldsymbol{H}=\boldsymbol{H}_{k}, \boldsymbol{W}=\boldsymbol{W}_{k}}\right)^{\mathrm{T}} \cdot \mathbf{Q}_{W} \boldsymbol{E}_{k}}{\Delta_{k}} \leqslant 1
\end{aligned}
$$

It is known that $\Delta_{k}>0$ from (65); therefore

$$
\begin{aligned}
-\Delta_{k}< & \left(\left.\frac{\partial \Delta}{\partial \boldsymbol{H}}\right|_{\boldsymbol{H}=\boldsymbol{H}_{k}, \boldsymbol{W}=\boldsymbol{W}_{k}}\right)^{\mathrm{T}} \cdot \mathbf{Q}_{H} \boldsymbol{E}_{k} \\
& +\left(\left.\frac{\partial \Delta}{\partial \boldsymbol{W}}\right|_{\boldsymbol{H}=\boldsymbol{H}_{k}, \boldsymbol{W}=\boldsymbol{W}_{k}}\right)^{\mathrm{T}} \cdot \mathbf{Q}_{W} \boldsymbol{E}_{k} \leqslant 0
\end{aligned}
$$

When the selection of the learning rates $\mathbf{Q}_{H}$ and $\mathbf{Q}_{W}$ satisfy (70), a convergent ILC law will be guaranteed.

\subsection{Iterative learning algorithm II}

In the previous iterative learning algorithm, the basis functions in the B-spline model are adjusted from 
batch to batch, which increases modelling accuracy progressively but also increases the computational load. To overcome this problem, an alternative way is to update the control sequence in the current batch from the PDF control errors in the previous batch without changing the model.

Again, taking the performance function in equation (62), the tracking error vector for the $k$ th iteration is defined as in equation (59). Following the iterative learning rule, the control input at the $i$ th time in the current batch should be updated based on the control input and tracking errors in the previous batch, i.e.

$$
u_{k+1, i}=u_{k, i}+\psi_{k+1}(i, i) E_{k}(i)
$$

where $\psi_{k+1}$ is the diagonal learning rate matrix for the $(k+1)$ th batch. The learning rates should be chosen to guarantee the asymptotical convergence of the algorithm, i.e.

$$
\sum_{i=1}^{m} \delta_{k, i} \leqslant \sum_{i=1}^{m} \delta_{k-1, i}
$$

Denoting the compensation term in (71) as

$$
\Delta u_{k, i}=\psi_{k+1}(i, i) E_{k}(i)
$$

condition (72) can be satisfied equivalently when

$$
\sum_{i=1}^{m} \frac{\partial \delta_{k, i}}{\partial \Delta u_{k-1, i-1}}<0
$$

Taking the PDF model (42) and (43) into $\delta_{k, i}$, the following equation can be derived

$$
\begin{aligned}
\frac{\partial \delta_{k, i}}{\partial \Delta u_{k-1, i-1}}= & 2 \int_{a}^{b} \overline{\boldsymbol{F}}_{k}(y) \Delta u_{k-1, i-1} \overline{\boldsymbol{F}}_{k}(y) \mathrm{d} y \\
& +2 \int_{a}^{b}\left[\overline{\boldsymbol{E}}_{k}(y) \mathrm{V}_{k, i-1}+\overline{\boldsymbol{F}}_{k}(y) u_{k-1, i-1}\right. \\
& \left.+\bar{g}_{k}(y)\right] \times \overline{\boldsymbol{F}}_{k}(y) \mathrm{d} y \quad(i \geqslant 2)
\end{aligned}
$$

where $\overline{\boldsymbol{E}}_{k}(y)=\mathbf{C}_{k}(y) \overline{\mathbf{A}}_{k}, \overline{\boldsymbol{F}}_{k}(y)=\mathbf{C}_{k}(y) \overline{\mathbf{B}}_{k}, \bar{g}_{k}(y)=L_{k}(y)-$ $g(y)$. Therefore, (74) turns out to be

$$
\begin{aligned}
& \sum_{i=1}^{m} \int_{a}^{b} \overline{\boldsymbol{F}}_{k}(y) \Delta u_{k-1, i-1} \overline{\boldsymbol{F}}_{k}(y) \mathrm{d} y \\
& \quad<-\sum_{i=1}^{m} \int_{a}^{b}\left[\overline{\boldsymbol{E}}_{k}(y) \boldsymbol{V}_{k, i-1}+\overline{\boldsymbol{F}}_{k}(y) u_{k-1, i-1}+\bar{g}_{k}(y)\right] \\
& \quad \times \overline{\boldsymbol{F}}_{k}(y) d y \quad(i \geqslant 1)
\end{aligned}
$$

Taking (73) into (76) gives

$$
\begin{aligned}
& \sum_{i=1}^{m} \int_{a}^{b} \overline{\boldsymbol{F}}_{k}(y) \psi_{k}(i-1, i-1) \overline{\boldsymbol{E}}_{k-1}(i-1) \overline{\boldsymbol{F}}_{k}(y) \mathrm{d} y \\
& \quad<-\sum_{i=1}^{m} \int_{a}^{b}\left[\overline{\boldsymbol{E}}_{k}(y) \boldsymbol{V}_{k, i-1}+\overline{\boldsymbol{F}}_{k}(y) u_{k-1, i-1}+\bar{g}_{k}(y)\right] \\
& \quad \times \overline{\boldsymbol{F}}_{k}(y) \mathrm{d} y
\end{aligned}
$$

The learning matrix $\psi_{k}$ should be designed to satisfy the condition in (77) for the asymptotical convergence of the closed-loop system.

\section{CASE STUDY OF MWD CONTROL}

The case for study is the semi-batch polystyrene process presented in section 2.1. To start with, the first-principle MWD model is developed to produce the dynamic MWD data and then the MWD data are used to set up the B-spline approximation model. The PDF control strategies are developed and implemented based on the B-spline model. The simulation conditions for the MWD system are given in Table 1. Comparisons are made between the two iterative learning PDF control strategies and the standard PDF control. In all the simulations, the initial MWD corresponds to the MWD under the monomer input ratio of $c=0.4$, and the target MWD is the one corresponding to $c=0.6$. A physical constraint of $0.4 \leqslant c \leqslant 0.8$ is considered for the monomer input ratio.

\subsection{MWD control by standard PDF control}

For a standard PDF control based on the fixed Bspline model, the control law is

$$
u_{i}=\frac{\int_{a}^{b} \mathbf{C}(y) \mathbf{D}_{0} \tilde{g}_{i}(y) \mathrm{d} y}{\int_{a}^{b}\left(\mathbf{C}(y) \mathbf{D}_{0}\right)^{2} \mathrm{~d} y+R}
$$

In order to obtain the necessary modelling accuracy, 10 third-order B-spline functions are selected for the MWD approximation. The standard PDF control was performed with $R=6 \times 10^{-6}$. The selection of $R$ is a trade-off: a larger value of $R$ increases the steady state MWD tracking error and a smaller $R$ causes more oscillations in the control input. The control input is shown in Fig. 4. The output MWDs are displayed in Fig. 5. The initial, final, and target MWDs are illustrated in Fig. 6. It can be seen that with the standard PDF control, the output MWD is 
Table 1 MWD system parameters

\begin{tabular}{ll}
\hline$K_{\mathrm{d}}$ & $9.48 \times 10^{16} \exp (-30798.5 / r T$ \\
$K_{\mathrm{i}}$ & $0.6 K_{d}$ \\
$K_{\mathrm{p}}$ & $6.306 \times 10^{8} \exp (-7067.8 / r T)$ \\
$K_{\mathrm{trm}}$ & $1.386 \times 10^{8} \exp (-12671.1 / r T)$ \\
$K_{\mathrm{t}}$ & $3.765 \times 10^{10} \exp (-1680 / r T)$ \\
$V$ & 3.927 \\
$F$ & 0.0286 \\
$T$ & 353 \\
$I^{0}$ & 0.0106 \\
$M^{0}$ & 4.81 \\
$r$ & 1.987 \\
$c$ & {$[0.4,0.8]$} \\
\hline
\end{tabular}

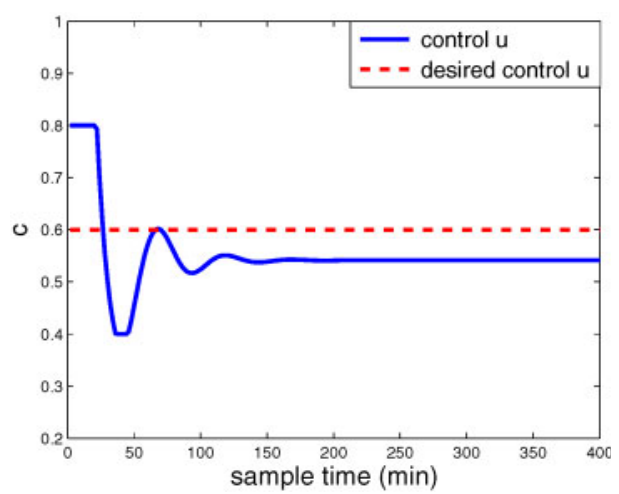

Fig. 4 Control input with standard PDF control

moved towards the target MWD, but the steady state tracking error remains when the control action is convergent.

\subsection{MWD control by iterative learning algorithm I}

In this ILC-PDF control simulation, five third-order B-spline functions are used in the MWD approximation. The interval in each batch is set to be 600 minutes. The simulation results are shown in Figs 7 to 10. It is observed from the simulation process that this control algorithm is computationally extensive

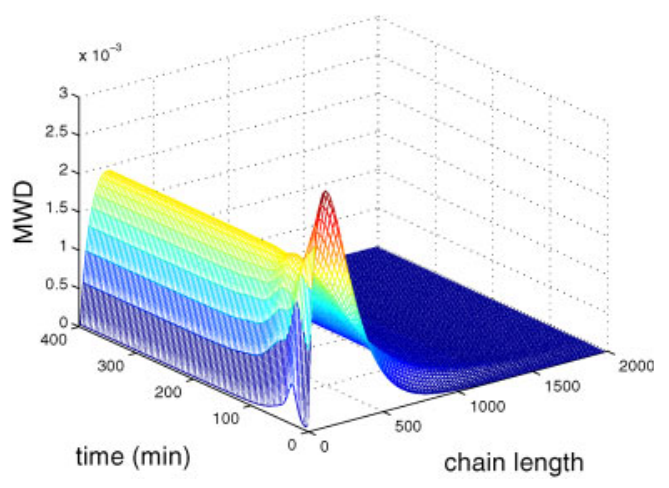

Fig. 5 MWD evolutions with standard PDF control

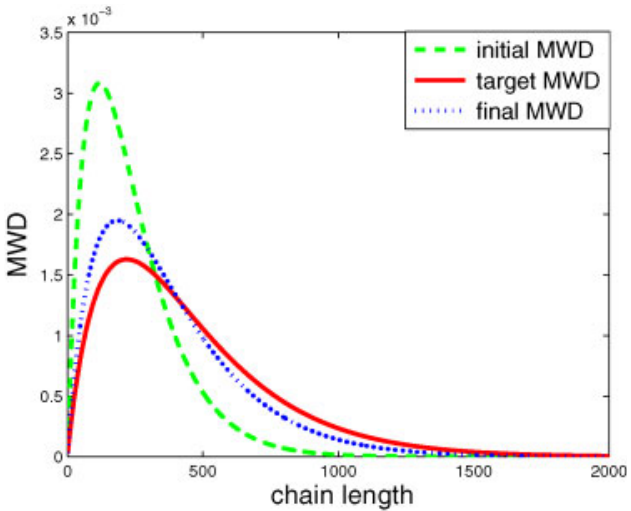

Fig. 6 Initial, target and final MWDs with standard PDF control

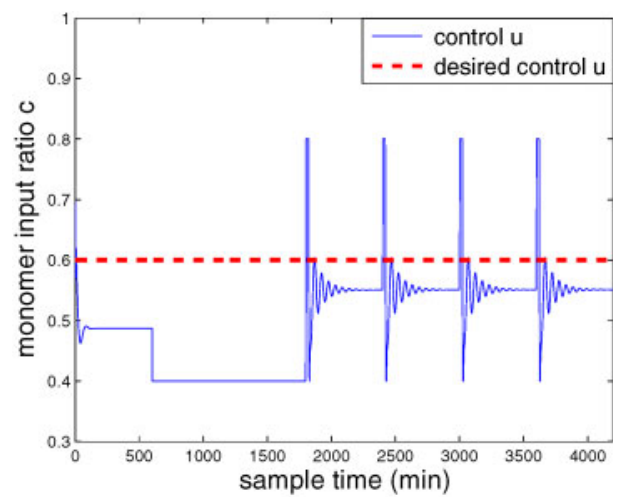

Fig. 7 Control input with the ILC-PDF algorithm I

since the B-splines need to be updated from batch to batch. Also, in order to satisfy the convergent condition in (70), the learning factors have to be adjusted several times in each batch, which further increases the computational effort. The results show that the distance between the output MWD and the target MWD can be reduced from batch to batch by the ILC strategy (see Fig. 8) with the reduced number of B-splines. However, the tuning ability is

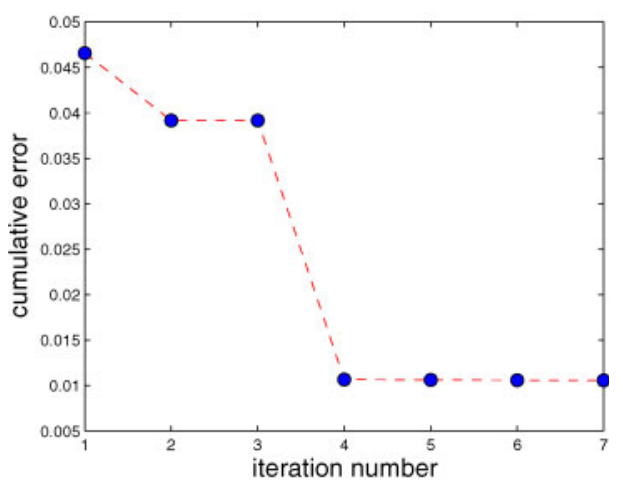

Fig. 8 Cumulative errors in each batch with the ILCPDF algorithm I 


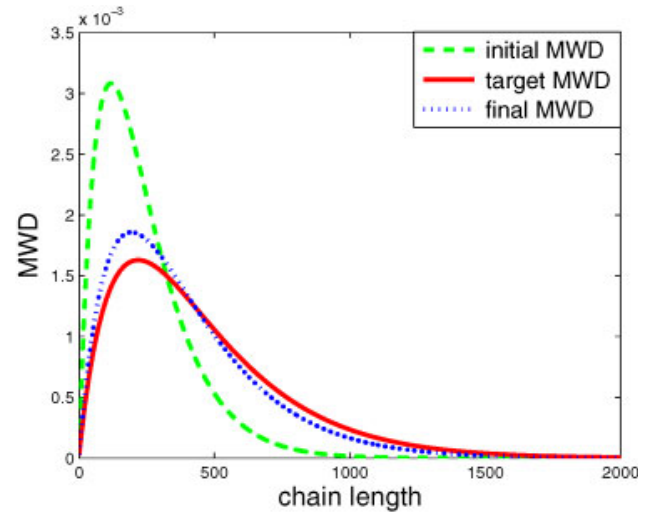

Fig. 9 Initial, target, and final MWDs with the ILCPDF algorithm I

saturated after several batches. This leaves the MWD tracking error at the end of the iterative learning cycle.

\subsection{MWD control by iterative learning algorithm II}

In this simulation, 10 third-order B-spline functions are selected for the MWD approximation. The interval length in each batch is set to be 400 minutes. The standard PDF control was implemented in the first batch with $R=6 \times 10^{-6}$ and then the iterative learning algorithm II was applied. Results are displayed in Figs 11 to 14. Although the steady state tracking errors remained in the first batch, the algorithm achieves a perfect tracking after another several batches. The cumulative error is decreasing from batch to batch.

Comparing the ILC-PDF controllers with the standard PDF controller, the ILC algorithm I uses a lower number of B-splines in modelling and it can achieve the decreasing tracking errors progressively.

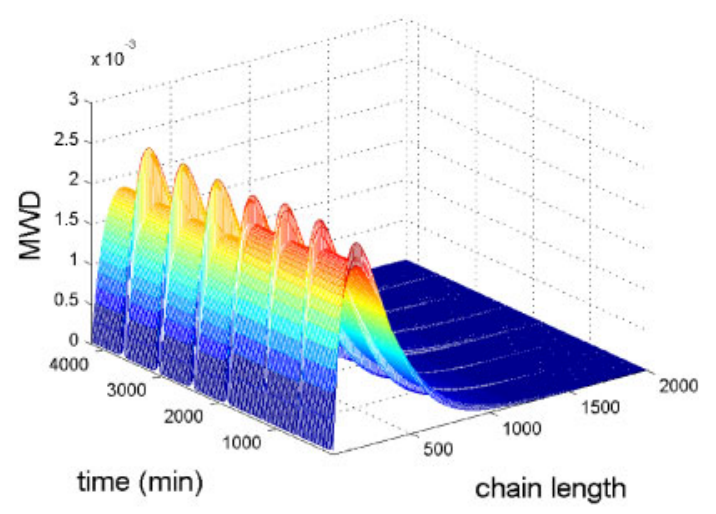

Fig. 10 Batch-to-batch MWD evolutions with the ILCPDF algorithm I

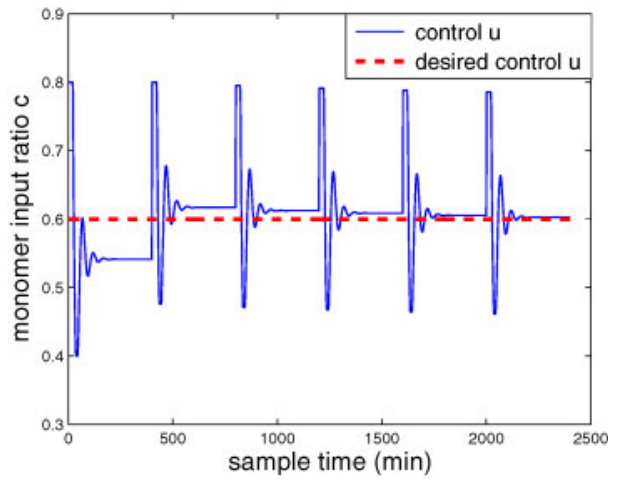

Fig. 11 Control input with the ILC-PDF algorithm II

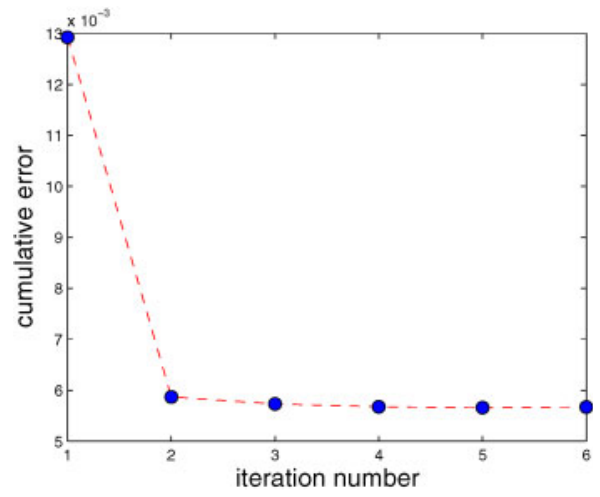

Fig. 12 Cumulative errors in each batch with the ILCPDF algorithm II

However, this algorithm is computationally expensive and, at least to this example, it could not eliminate the MWD tracking errors at the end of the control cycle. In the second algorithm, the model is updated in each batch and then the standard PDF control strategy is applied based on the updated model. The convergence condition is given to guarantee the decrease of MWD tracking errors from batch to batch. Nevertheless, the improvement of

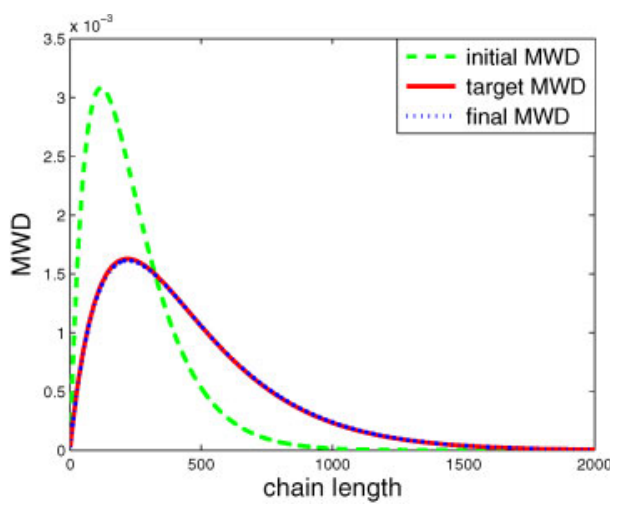

Fig. 13 Initial, target, and final MWDs with the ILCPDF algorithm II 


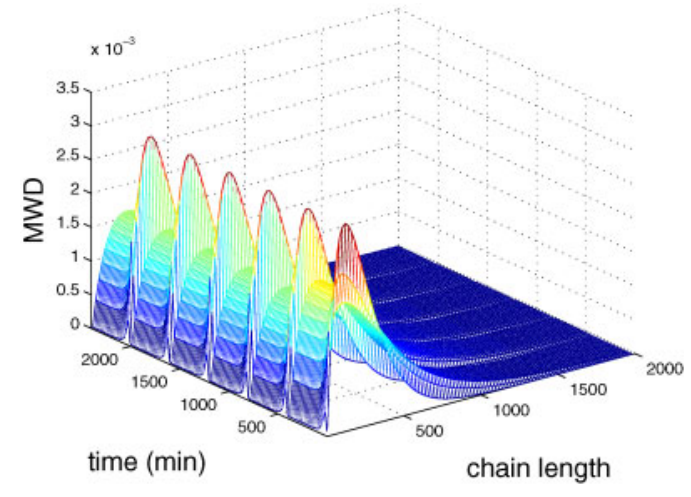

Fig. 14 Batch-to-batch MWD evolutions with the ILCPDF algorithm II

the modelling accuracy cannot be guaranteed by the current tuning principle and therefore the tracking errors may still remain after the tuning is convergent. By employing the second iterative learning algorithm, the B-spline model does not need to be updated during the process and the control input in the current batch is adjusted according to the MWD tracking errors in the previous batch. This is a simple solution compared with the first ILC-PDF control algorithm, but it can achieve perfect MWD tracking after several batches. The function of batch-to-batch tuning based on the previous tracking errors is equivalent to the integral efforts in feedback control and is therefore capable of eliminating tracking errors. This means that the fundamental principle of iterative learning control enables performance improvement in output PDF control.

\section{CONCLUSIONS}

The challenging problem of feedback MWD control has been studied in this work. It is different from most of the current control strategies on MWD shaping because the complete MWD is controlled as a closed-loop variable under the framework of output PDF control. Iterative learning strategies have been attempted to improve the control quality. It can be seen from the case study that even for a simulated MWD system, the model is highly complex and it is difficult to achieve the perfect PDF tracking when using linear B-spline models and employing the standard output PDF control strategy. Introducing the idea of iterative learning control into output PDF control does improve the control performance in that it can reduce the PDF control errors from batch to batch. The present methods are developed based on B-spline models which becomes computationally challenging when the system is complicated. It is worth further efforts in exploring different modelling methodology that would be more convenient for online iterative learning control. To provide more benefit from the ILC's advantages, investigations on developing efficient learning algorithms in both modelling and control are required in future work.

\section{ACKNOWLEDGEMENT}

The authors would like to thank Professor Liulin Cao from Beijing University of Chemical Technology for her great help in MWD model development. This work was supported by Chinese NSFC 307705660 and 60534010 and the 111 Project (B08015) with the Northeastern University of China which the second author is affiliated to. These are gratefully acknowledged.

\section{REFERENCES}

1 Crowley, T. J. and Choi, K. Y. Experimental studies on optimal molecular weight distribution control in a batch-free radical polymerization process. Chem. Engng Sci., 1998, 53(15), 2769-2790.

2 Takamatsu, T., Shioya, S., and Okada, Y. Molecular weight distribution control in a batch polymerization reactor. Ind. Engng Chem. Res., 1988, 27(1), 93-99.

3 Kammona, O., Chatzi, E. G., and Kiparissides, C. Recent developments in hardware sensors for the on-line monitoring of polymerization reactions. $J$. Macromolec. Sci., Rev. Macromolec. Chem. Phys., 1999, C39(1), 57C134.

4 Pinto, J. C. A matrix representation of polymer chain size distributions, 1. Linear polymerization mechanisms at steady-state conditions. Macromolec. Theory Simulation, 2001, 10(2), 79-99.

5 Nele, M., Sayer, C., and Pinto, J. C. Computation of molecular weight distributions by polynomial approximation with complete adaptation procedures. Macromolec. Theory Simulations, 1999, 8(3), 199-213.

6 Sayer, C., Aranjo, P. H. H., Arzamendi, G., Asua, J. M., Elma, E. L., and Pinto, J. C. Modeling molecular weight distribution in emulsion polymerization reactions with transfer to polymer. J. Polym. Sci. A: Polym. Chem., 2001, 39(20), 3513-3528.

7 Crowley, T. J. and Choi, K. Y. Calculation of molecular weight distribution from molecular weight moments in free radical polymerisation. Ind. Engng Chem. Res., 1997, 36(5), 1419-1423.

8 Christov, L. and Georgiev, G. An algorithm for determination of the copolymer molecular weight distribution by Markov chain simulation. Macromolec. Theory Simulations, 1995, 4(1), 177-193. 
9 Storti, G., Polotti, G., Cociani, M., and Morbidelli, M. Molecular weight distribution in emulsion polymerization. I. the homopolymer case. J. Polym. Sci. A: Polym. Chem., 1992, 30, 731-750.

10 Storti, G., Polotti, G., Cociani, M., and Morbidelli, M. Molecular weight distribution in emulsion polymerization. II. The copolymer case. J. Polym. Sci. A: Polym. Chem., 1992, 30, 751-777.

11 Flory, P. J. Principles of polymer chemistry, 1953 (Cornell University Press, Ithaca, New York).

12 Soares, J. B. P. and Hamielec, A. E. Deconvolution of chain-length distributions of linear polymers made by multiple-site-type catalysts. Polymer, 1995, 36(11), 2257-2263.

13 Soares, J. B. P., Kim, J. D., and Rempel, G. L. Analysis and control of the molecular weight and chemical composition distributions of polyolefins made with metallocene and Ziegler-Natta catalysts. Ind. Engng Chem. Res., 1997, 36(4), 1144-1150.

14 Stockmayer, W. H. Distribution of chain lengths and compositions in copolymers. J. Chem. Phys., 1945, 13, 199.

15 Cao, L. and Yue, H. Mathematical models of microscopic quality for polybutadiene production. J. Beijing Inst. Chem. Technol., 1994, 21 (2), 65-69.

16 Yue, H. and Cao, L. Reaction extent modeling of a butadiene polymetization process. In Proceedings of the IFAC International Symposium on Advanced control of chemical processes, Pisa, Italy, 14-16 June 2000, pp. 1043-1048.

17 Cao, L. and Lu, N. Modelling of a continuous PET process. J. System Simulation, 2001, 13(4), 536-538.

18 Yue, H., Wang, H., and Cao, L. Control oriented Bspline modelling of a dynamic MWD-system. In Proceedings of the IFAC International, Symposium on Advanced control of Chemical Processes, Gramado, Brazil, 2-5 April 2006, pp. 719-724.

19 Nele, M. and Pinto, J. C. Molecular-weight multimodality of multiple Flory distributions. Macromolec. Theory Simulation, 2002, 11(3), 293-307.

20 Salazar, A., Gugliotta, L. M., Vega, J. R., and Meira, G. R. Molecular weight control in a starved emulsion polymerization of styrene. Ind. Engng Chem. Res., 1998, 37(9), 3582-3591.

21 Kiparissides, C. Challenges in particulate polymerization reactor modeling and optimization: a population balance perspective. J. Process Control, 2006, 16(3), 205-224.

22 Yue, H., Wang, H., and Zhang, J. F. Modelling and control of molecular weight distribution in polymerization processes. Control Instrum. Chem. Industry, 2004, 31(6), 1-7.

23 Ellis, M. F., Taylor, T. W., and Jensen, K. F. On-line molecular weight distribution estimation and control in batch polymerization. Am. Inst. Chem. Engrs J., 1994, 40(3), 445-462.

24 Kiparissides, C., Seferlis, P., Mourikas, G., and Morris, A. J. Online optimizing control of molecular weight properties in batch free-radical poly- merization reactors. Ind. Engng Chem. Res., 2002, 41(24), 6120-6131.

25 Alhamad, B., Romagnoli, J. A., and Gomes, V. G. Advanced modelling and optimal operating strategy in emulsion copolymerization: application to styrene/MMA system. Chem. Engng Sci., 2005, 60(10), 2795-2813.

26 Alhamad, B., Romagnoli, J. A., and Gomes, V. G. On-line multi-variable predictive control of molar mass and particle size distributions in free-radical emulsion copolymerization. Chem. Engng Sci., 2005, 60(23), 6596-6606.

27 Chang, J.-H. and Liao, P.-H. Molecular weight control of a batch polymerisation reactor: experimental study. Ind. Engng Chem. Res., 1999, 38(1), 144-153.

28 Clarke-Pringle, T. L. and MacGregor, J. F. Optimization of molecular-weight distribution using batch-to-batch adjustments. Ind. Engng Chem. Res., 1998, 37(9), 3660-3669.

29 Crowley, T. J. and Choi, K. Y. Discrete optimal control of molecular weight distribution in a batch free radical polymerization process. Ind. Engng Chem. Res., 1997, 36(9), 3676-3684.

30 Echevarria, A., Leiza, J. R., de la Cal, J. C., and Asua, J. M. Molecular weight distribution control in emulsion polymerisation. Am. Inst. Chem. Engrs J., 1998, 44(7), 1667-1679.

31 Vicente, M., BenAmor, S., Gugliotta, L. M., Leiza, J. R., and Asua, J. M. Control of molecular weight distribution in emulsion polymerization using online reaction calorimetry. Ind. Engng Chem. Res., 2001, 40(1), 218-227.

32 Vicente, M., Sayer, C., Leiza, J. R., Arzamendi, G., Lima, E. L., Pinto, J. C., and Asua, J. M. Dynamic optimization of non-linear emulsion copolymerization systems open-loop control of composition and molecular weight distribution. Chem. Engng J., 2002, 85(2-3), 339-349.

33 Wang, H. Bounded dynamic stochastic systems: modelling and control, 2000 (Springer-Verlag, London).

34 Crowley, T. J., Meadows, E. S., Kostoulas, E., and Doyle III, F. J. Control of particle size distribution described by a population balance model of semibatch emulsion polymerization. J. Process Control, 2000, 10(5), 419-432.

35 Flores-Cerrillo, J. and MacGregor, J. F. Control of particle size distributions in emulsion semibatch polymerization using mid-course correction polices. Ind. Engng Chem. Res., 2002, 41(7), 1805-1814.

36 Immanuel, C. D. and Doyle III, F. J. Open-loop control of particle size distribution on semi-batch emulsion copolymerisation using a genetic algorithm. Chem. Engng Sci., 2002, 57(20), 4415-4427.

37 Braatz, R. D. Advanced control of crystallization processes. Annual Rev. Contr., 2002, 26(1), 87-99.

38 Eek, R. A. and Bosgra, O. H. Controllability of particulate processes in relation to the sensor 
characteristics. Powder Technol., 2000, 108(2-3), 137-146.

39 Gommeren, H. J. C., Heitzmann, D. A., Moolenaar, J. A. C., and Scarlett, B. Modelling and control of a jet mill plant. Powder Technol., 2000, 108(2-3), 147-154.

40 Ma, D. L., Tafti, D. K., and Braatz, R. D. Optimal control and simulation of multidimensional crystallization processes. Comput. Chem. Engng, 2002, 26(7-8), 1103-1116.

41 Smook, G. A. Handbook for Pulp and Paper Technologists, 1998 (Angus Wilde Publications, Vancouver, British Columbia).

42 Sun, X. B., Yue, H., and Wang, H. Modelling and control of the flame temperature distribution using probability density function shaping. Trans. Inst. Measmt and Control, 2006, 28(5), 401-428.

43 Wang, H. Control of the output probability density functions for a class of nonlinear stochastic systems. In Proceedings of the IFAC Workshop on Algorithms and architectures for real-time control, Cancun, Mexico, 15-17 April 1998, pp. 95-99.

44 Wang, H. Robust control of the output probability density functions for multivariable stochastic systems. IEEE Trans. Autom. Control, 1999, 44(11), 2103-2107.

45 Arimoto, A., Kawamura, S., and Miyazaki, F. Bettering operation of robots by learning. J. Robolic Systems, 1984, 1(2), 123-140.

$46 \mathrm{Xu}$, J. and Tan, Y. F. (Eds) Linear and nonlinear iterative learning control, vol.291, of Lecture notes in control and information science, 2003 (SpringerVerlag, Berlin, Germany).

47 Svante, G. and Mikael, N. On the design of ILC algorithms using optimization. Automatica, 2001, 37(12), 2011-2016.

$48 \mathrm{Xu}$, J. X. and Tan, Y. Robust optimal design and convergence properties analysis of iterative learning control approaches. Automatica, 2002, 38(11), 1867-1880.

49 Wang, H. and Yue, H. Iterative B-spline neural networks for stochastic distribution control and its application in industrial process. IEEE J. Intell. Cybernetics Systems, 2005, 2.

50 Wang, H., Zhang, J. F., and Yue, H. Iterative learning control of output PDF shaping in stochastic systems. In Proceedings of the 2005 IEEE International Symposium on Intelligent control, Limassol, Cyprus, 27-29 June 2005, pp. 1225-1230.

51 Afshar, P., Yue, H., and Wang, H. Robust iterative learning control of output PDF in non-Gaussian stochastic systems using Youla parametrization. In Proceedings of the 2007 American Control Conference, New York, 11-13 July 2007, pp. 576-581.

52 Wang, H., Afshar, P., and Yue, H. ILC-based generalised PI control for output PDF of stochastic systems using LMI and RBF neural networks. In Proceedings of the 45th IEEE Conference on decision and control, San Diego, California, 13-15 December 2006, pp. 5048-5053.
53 Angerman, H. J. The phase behavior of polydisperse multiblock copolymer melts: a theoretical study, http://irs.ub.rug.nl/ppn/166955302, 1998.

\section{APPENDIX}

\section{Notation}

$c$

F

$F_{\mathrm{I}}$

$F_{\mathrm{M}}$

$h$

$I$

$I^{0}$

$K_{\mathrm{d}}$

$K_{\mathrm{i}}$

$K_{\mathrm{p}}$

$K_{\mathrm{t}}$

$K_{\text {trm }}$

$M$

$M^{0}$

$M_{n}$

$n$

$P$

$P_{j}$

R

$R^{*}$

$R_{j}$

$U_{k}$

V

$Z_{k}$

$\Gamma$

$\theta$

$\mu \quad$ mean of the distribution

$\sigma^{2} \quad$ variance of the distribution monomer input ratio total input flowrate $\left(\mathrm{L} \cdot \mathrm{min}^{-1}\right)$ initiator input flowrate $\left(\mathrm{L} \cdot \mathrm{min}^{-1}\right)$ monomer input flowrate $\left(\mathrm{L} \cdot \mathrm{min}^{-1}\right.$ ) parameter of the Schultz-Zimm distribution initiator and its concentration $\left(\mathrm{mol} \cdot \mathrm{L}^{-1}\right)$

initial concentration of the initiator in the input flow $\left(\mathrm{mol} \cdot \mathrm{L}^{-1}\right)$

initiator decomposition rate constant $\left(\min ^{-1}\right)$

initiation reaction constant $\left(\mathrm{L} \cdot \mathrm{mol}^{-1} \cdot \mathrm{min}^{-1}\right)$

propagation rate constant $\left(\mathrm{L} \cdot \mathrm{mol}^{-1} \cdot \mathrm{min}^{-1}\right)$ termination rate constant $\left(\mathrm{L} \cdot \mathrm{mol}^{-1} \cdot \mathrm{min}^{-1}\right)$

chain transfer rate constant $\left(\mathrm{L} \cdot \mathrm{mol}^{-1} \cdot \mathrm{min}^{-1}\right)$

monomer and its concentration $\left(\mathrm{mol} \cdot \mathrm{L}^{-1}\right)$

initial concentration of the initiator in the input flow $\left(\mathrm{mol} \cdot \mathrm{L}^{-1}\right)$ number average chain length chain length total concentration of the dead polymers $\left(\mathrm{mol} \cdot \mathrm{L}^{-1}\right)$

dead polymer with chain length $j$ or its concentration $\left(\mathrm{mol} \cdot \mathrm{L}^{-1}\right)$

total concentration of the radicals $\left(\mathrm{mol} \cdot \mathrm{L}^{-1}\right)$

primary radical

active polymer radical with chain length $j$ or its concentration $\left(\mathrm{mol} \cdot \mathrm{L}^{-1}\right)$

moments of radicals

volume of reaction mixture (L)

moments of polymers

Gamma function

average residential time of reactants

in the reactor (min) 